\title{
AN OSCILLATION THEOREM FOR SUBLINEAR ELLIPTIC DIFFERENTIAL INEQUALITIES
}

\author{
NORIO YOSHIDA
}

\begin{abstract}
Sublinear elliptic differential inequalities with variable coefficients are studied. Sufficient conditions are given that all solutions are oscillatory in exterior domains. Riccati inequalities are used to establish sublinear oscillation criteria.
\end{abstract}

Oscillation of solutions of sublinear elliptic differential equations has been discussed by several authors, see e.g. $[2,3,5,6,8,9]$ and the references contained therein. In particular, sublinear elliptic differential operators with variable coefficients have been studied by the author [9]. In [5] Noussair and Swanson established an oscillation criterion by using Riccati transformations. The purpose of this paper is to solve an open question stated in [5, p.922, (3)] by employing a Riccati inequality.

We are concerned with the oscillatory behaviour of solutions of the sublinear elliptic differential operator $L$ defined by

$$
L[u] \equiv \sum_{i, j=1}^{n} D_{i}\left(a_{i j}(x) D_{j} u\right)+c(x, u), \quad x \in \Omega,
$$

where $\Omega$ is an exterior domain in $R^{n}$, i.e. $\Omega$ contains the complement of some $n$-ball in $R^{n}$. Points in $R^{n}$ will be denoted by $x=\left(x_{1}, \ldots, x_{n}\right)$, and differentiation with respect to $x_{i}$ by $D_{i}$,

Received 16 May 1984.

Copyright Clearance Centre, Inc. Serial-fee code: 0004-9727/84 $\$ A 2.00+0.00$. 
$i=1, \ldots, n$. The notation $|x|$ will be used for the Euclidean length of $x \in R^{n}$. The domain $D_{L}(\Omega)$ of $L$ is defined to be the set of all realvalued functions of class $C^{2}(\Omega)$. We assume that the following conditions hold throughout this paper:

(A-I) $c(x, \xi)$ is a real-valued continuous function in $\Omega \times R^{1}$;

(A-II) $\quad D_{k} D_{\ell} a_{i j}(x)$ are locally uniformly Hölder-continuous in $\Omega$

$$
(i, j, k, l=1, \ldots, n) \text {; }
$$

(A-III) the matrix $A(x) \equiv\left(a_{i j}(x)\right)_{i, j=1}^{n}$ is real symmetric and strictly positive definite in $\Omega$ (ellipticity condition); (A-IV) $c(x, \xi) \geqq p_{1}(x) \phi_{1}(\xi)$ for all $(x, \xi) \in \Omega \times[0, \infty)$, where $p_{1}$ is continuous in $\Omega, \phi_{1} \in C^{1}(0, \infty)$ and $\phi_{1}(\xi)>0$ in $(0, \infty)$;

(A-V) $\quad c(x, \xi) \leqq-p_{2}(x) \phi_{2}(-\xi)$ for all $(x, \xi) \in \Omega \times(-\infty, 0)$, where $p_{2}$ is continuous in $\Omega, \phi_{2} \in C^{1}(0, \infty)$ and $\phi_{2}(\xi)>0$ in

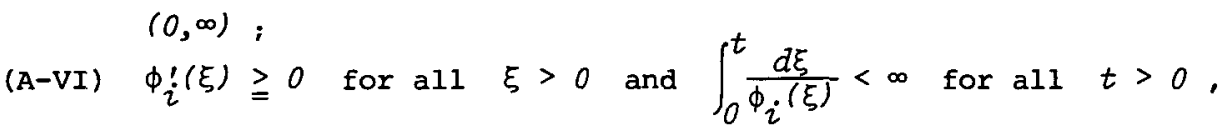
$i=1,2$.

The $n$-dimensional sublinear Enden-Fowler equation

$$
\sum_{i, j=1}^{n} D_{i}\left(a_{i j}(x) D_{j} u\right)+p(x)|u|^{\gamma_{\text {sgn }}} u=0,0<\gamma<1,
$$

is an important special case of $L[U]=0$ which satisfies $(A-I V)-(A-V I)$.

DEFINITION. A function $u \in D_{L}(\Omega)$ is said to be oscillatory in $\Omega$ if it has arbitrarily large zeroes. The inequality $u L[u] \leqq 0$ is said to be oscizzatory in $\Omega$ whenever every solution $u$ of the inequality is oscillatory in $\Omega$.

Since $\Omega$ is an exterior domain in $R^{n}$, there exists a number $\varepsilon>0$ such that $R^{n}(\varepsilon) \subset \Omega$, where $R^{n}(\varepsilon) \equiv\left\{x \in R^{n}:|x|>\varepsilon\right\}$. Let $x_{0}$ be a fixed point in $R^{n}(\varepsilon)$. Then, there exists a fundamental solution 
$E(x) \in C^{2}\left(R^{n}(\varepsilon)\left\{x_{0}\right\}\right)$ of the operator $P \equiv \sum_{i, j=1}^{n} D_{i} a_{i j}(x) D_{j}$ with a singularity at the point $x_{0}$ (see Itô $[1$, p.84]). We assume that $\lim _{|x|+\infty} E(x)=-\infty(n=2)$ and $\lim _{|x|+\infty} E(x)=0(n \geqq 3)$. In the case that $P=\Delta \quad$ (Laplacian), one takes $E(x)=(2 \pi)^{-1} \log \left(|x|^{-1}\right)(n=2)$ and $E(x)=\left(\sigma_{n}(n-2)\right)^{-1}|x|^{2-n}(n \geqq 3)$, where $\sigma_{n}$ denotes the surface area of the unit sphere in $R^{n}$. We define

$$
\rho(x)= \begin{cases}\exp (-2 \pi E(x)), & n=2, \\ \left(\sigma_{n}(n-2) E(x)\right)^{1 /(2-n)}, & n \geqq 3 .\end{cases}
$$

We note that $\rho(x)=|x|$ if $P=\Delta$. The distance of each point on $S_{r} \equiv\{x \in \Omega: \rho(x)=r\}$ from the origin tends to infinity as $r \rightarrow \infty$, and therefore there exists a number $r_{0}>0$ such that $\left\{x \in R^{n}: \rho(x)>r_{0}\right\} \subset R^{n}(\varepsilon) \subset \Omega$. We find that each level surface $S_{p}$ represents a smooth closed $n-1$ dimensional surface in $\Omega$ and $\Omega\left(r_{1}, r_{2}\right) \equiv\left\{x \in \Omega: r_{1}<\rho(x)<r_{2}\right\}\left(r_{0}<r_{1}<r_{2}\right)$ is a bounded domain with boundary $S_{r_{1}} \cup S_{r_{2}}$ (cf. Levine and Payne [4]).

Associated with every positive function $u \in D_{L}(\Omega)$, we define the $n$-vector function $w(x)$ by

$$
w(x)=-\frac{1}{\phi_{1}(u)} A(x) \nabla u,
$$

where $\nabla u$ denotes the gradient of $u$. The following notation will be used:

$$
\begin{aligned}
& p(x)=\min \left\{p_{1}(x), p_{2}(x)\right\}, x \in \Omega, \\
& \tilde{p}(x)=\left(\sigma_{n^{2}} x^{n-1}\right)^{-1} \int_{S_{r}} p(x)|\nabla \sigma|^{-1} d \sigma .
\end{aligned}
$$


By the same arguments as were used by Noussair and Swanson [5], we obtain the following result.

THEOREM. Under assumptions $(A-I)-(A-V I)$, the sublinear elliptic differential inequality $u L[u] \leqq 0$ is oscillatory in an exterior domain $\Omega$ of $R^{n}$ if

$$
\lim \sup \int_{r \rightarrow \infty}^{r}\left(1-\frac{\theta_{n}(t)}{\theta_{n}(r)}\right) \tilde{t p}(t) d t=\infty
$$

for some $\tilde{r}>r_{0}$, where $\theta_{2}(r)=\log r$ and $\theta_{n}(r)=r^{n-2}(n \geqq 3)$.

Proof. Suppose to the contrary that there exists a solution $u$ of $u L[u] \leqq 0$ which has no zero in $\Omega\left(r_{1}, \infty\right)$ for some $r_{1}>r_{0}$. First we assume that $u>0$ in $\Omega\left(r_{1}, \infty\right)$. It follows from a result of Noussair and Swanson [5, Lemma 1] that

$$
\operatorname{div} w(x) \geqq p_{1}(x)
$$

Integrating (3) over $\Omega\left(r_{1}, r\right)$, we obtain

$$
\int_{\Omega\left(r_{1}, r\right)} \operatorname{div} w(x) d x \geqq \int_{\Omega\left(r_{1}, r\right)} p_{1}(x) d x \geqq \int_{\Omega\left(r_{1}, r\right)} p(x) d x .
$$

An application of the Divergence Theorem shows that

$$
\int_{S_{r}} w^{*}(\nabla \rho)|\nabla \rho|^{-1} d \sigma-\int_{S_{r_{1}}} w^{*}(\nabla \rho)|\nabla \rho|^{-1} d \sigma \geqq \int_{\Omega\left(r_{1}, r\right)} p(x) d x,
$$

where * denotes the transpose. We define

$$
Q[u](r) \equiv \int_{S_{r}}(\nabla G(u)) * A(x)(\nabla \rho)|\nabla \rho|^{-1} d \sigma,
$$

where $G(u)=\int_{0}^{u} \phi_{1}(\xi)^{-1} d \xi$. Since

$w^{*}(\nabla \rho)|\nabla \rho|^{-1}=-\phi_{1}(u)^{-1}(\nabla u)^{*} A(x)(\nabla \rho)|\nabla \rho|^{-1}=-(\nabla G(u)) * A(x)(\nabla \rho)|\nabla \rho|^{-1}$, we have 
Sublinear elliptic differential inequalities

$$
Q[u](r)=-\int_{S_{r}} w^{*}(\nabla \rho)|\nabla \rho|^{-1} d \sigma
$$

Hence, (4) is equivalent to

$$
Q[u](r)-Q[u]\left(r_{1}\right) \leqq-\int_{\Omega\left(r_{1}, r\right)} p(x) d x .
$$

We easily obtain

$$
\int_{\Omega\left(r_{1}, r\right)} p(x) d x=\sigma_{n} \int_{r_{1}}^{r} t^{n-1} d t\left(\left(\sigma_{n} t^{n-1}\right)^{-1} \int_{S_{t}} p(x)|\nabla \rho|^{-1} d \sigma\right)
$$

$$
=\sigma_{n} \int_{r_{1}}^{r} t^{n-1} \tilde{p}(t) d t
$$

Hence, it follows from (5) and (6) that

$$
Q[u](r)-Q[u]\left(r_{1}\right) \leqq-\sigma_{n} \int_{r_{1}}^{r} t^{n-1} \tilde{p}(t) d t,
$$

and therefore

(7) $\int_{r_{1}}^{r} \frac{Q[u](n)}{\sigma_{n} n^{n-1}} d n-\int_{r_{1}}^{r} \frac{Q[u]\left(r_{1}\right)}{\sigma_{n} n^{n-1}} d n \leqq-\int_{r_{1} n^{n-1}}^{r} \int_{r_{1}}^{n} t^{n-1} \tilde{p}(t) d t$.

Using a result of Suleimanov [7, Lemma 2], we obtain

$$
\frac{Q[u](r)}{\sigma_{n} n^{n-1}}=\frac{d}{d n} M[u](n)
$$

where

$$
M[u](\eta)=\left(\sigma_{n} n^{n-1}\right)^{-1} \int_{S_{n}} G(u)(\nabla \rho) * A(x)(\nabla \rho)|\nabla \rho|^{-1} d \sigma,
$$

(cf. the author [9, the proof of Lemma 2.1]). It is easy to see that 


$$
\int_{r_{1}}^{r} \frac{d n}{n^{n-1}} \int_{r_{1}}^{n} t^{n-1} \tilde{p}(t) d t=\int_{r_{1}}^{r} t^{n-1} \tilde{p}(t) d t \int_{t}^{r} \frac{d n}{n^{n-1}}
$$

$$
=k_{n}(r) \int_{r_{1}}^{r} t \tilde{p}(t)\left(1-\frac{\theta_{n}(t)}{\theta_{n}(r)}\right) d t
$$

where $k_{2}(r)=\log r$ and $k_{n}(r)=(n-2)^{-1}(n \geqq 3) \cdot$ Combining $(7)-(9)$ yields

$$
\begin{gathered}
M[u](r)-M[u]\left(r_{1}\right)+Q[u]\left(r_{1}\right) \sigma_{2}^{-1} \log r_{1} \\
\leqq-(\log r)\left(\int_{r_{1}}^{r}\left(1-\frac{\theta_{2}(t)}{\theta_{2}(r)}\right) t \tilde{p}(t) d t-\sigma_{2}^{-1} Q[u]\left(r_{1}\right)\right)(n=2),
\end{gathered}
$$

and

$$
\begin{gathered}
M[u](r)-M[u]\left(r_{1}\right)-Q[u]\left(r_{1}\right) \sigma_{n}^{-1}(n-2)^{-1} r_{1}^{-n+2} \\
\leqq-(n-2)^{-1}\left(\int_{r_{1}}^{r}\left(1-\frac{\theta_{n}(t)}{\theta_{n}(r)}\right) t \tilde{p}(t) d t+\sigma_{n}^{-1} Q[u]\left(r_{1}\right) r^{-n+2}\right)(n \geqq 3) .
\end{gathered}
$$

Hence, condition (2) implies that the right hand side of (10) and (11) are not bounded from below, and consequently $M[u](r)$ would take negative values for sufficiently large $r$. This contradicts the positivity of $M[u](r)$.

If $u<0$ in $\Omega\left(x_{1}, \infty\right), v \equiv-u$ is a positive solution of $v(-L[-v]) \leqq 0$. In view of the assumption $(A-V)$ we conclude that

$$
\begin{aligned}
\sum_{i, j=1}^{n} D_{i}\left(a_{i, j}(x) D_{j} v\right)+p_{2}(x) \phi_{2}(v) & \leqq \sum_{i, j=1}^{n} D_{i}\left(a_{i j}(x) D_{j} v\right)-c(x,-v) \\
& =-L[-v] \leqq 0 .
\end{aligned}
$$

Hence, $v$ is a positive solution of (12). Repeating the same arguments as in the case where $u>0$, we are led to a contradiction. This completes the proof. 
The proof of the following result is quite similar to that of Corollary of the author $[9$, p. 716], and hence will be omitted.

COROLLARY. Under assumptions $(A-I)-(A-V I)$, the sublinear elliptic differential inequality $u L[u] \leqq 0$ is oscillatory in an exterior domain $\Omega$ of $R^{n}$ if

$$
\int_{\tilde{r}}^{\infty} t \tilde{p}(t) d t=\infty \text { for some } \tilde{r}>r_{0} .
$$

REMARK. In the case where $n=2, \phi_{1} \equiv \phi_{2}$ and $a_{i j}(x)=\delta_{i j}$ (the Kronecker delta), Corollary reduces to a result of Noussair and Swanson [5, Theorem 15]. Let $\Phi(\xi)$ be defined by

$$
\Phi(\xi)= \begin{cases}\phi_{1}(\xi) & \text { for } \xi \geqq 0, \\ -\phi_{2}(-\xi) & \text { for } \xi<0,\end{cases}
$$

where $\phi_{1}(0)=\lim _{\xi \rightarrow+0} \phi_{2}(\xi)=0$. If $p_{1}(x)=p_{2}(x)=p(x)$ in $\Omega$ and $c(x, \xi)=p(x) \Phi(\xi)$, then Theorem reduces to a result of the author [9, Theorem 4.1]. For the sublinear equation $\Delta u+p(x) \Phi(u)=0$ oscillation criteria (2) and (13) were established by Kura [3] and Kitamura and Kusano [2], respectively.

\section{References}

[1] Seizô Itô, "Fundamental solutions of parabolic differential equations and boundary value problems", Japan. J. Math. 27 (1957), 55-102.

[2] Yuichi Kitamura and Takaŝi Kusano, "An oscillation theorem for a sublinear Schrödinger equation", Utilitas Math. 14 (1978), 171-175.

[3] Takeshi Kura, "Oscillation criteria for a class of sublinear elliptic equations of the second order", Utilitas Math. 22 (1982), 335-341. 
[4] Howard A. Levine and Lawrence E. Payne, "On the nonexistence of entire solutions to nonlinear second ordex elliptic equations", SIAM J. Math. Anal. 7 (1976), 337-343.

[5] Ezzat S. Noussair and Charles A. Swanson, "Oscillation of semilinear elliptic inequalities by Riccati transformations", Canad. $J$. Math. 32 (1980), 908-923.

[6] Hiroshi Onose, "Oscillation criteria for sublinear Schrödinger equation", Proc. Amer. Math. Soc. 85 (1982), 69-72.

[7] N. M. Suleimanov, "On the behaviour of solutions of second order nonlinear elliptic equations with linear principal part", Dokl. Akad. Nauk SSSR, 247 (1979), 805-809.

[8] Charles A. Swanson, "Semilinear second order elliptic oscillation", Canad. Math. Bull. 22 (1979), 139-157.

[9] Norio Yoshida, "Oscillation properties of solutions of second order elliptic equations", SIAM J. Math. Anal. 14 (1983), 709-718.

Department of Mathematics,

Faculty of Engineering,

Iwate University,

Morioka, Japan. 